\title{
0 Falso Dilema sobre a Luta Antivetorial e as Perspectivas de Controle da Doença de Chagas no Brasil. BHC ou BNH?
}

\section{The False Dilemma about Antivectorial Strategies and Possibilities for Controlling Chagas' Disease in Brazil. BHC or BNH?}

\author{
José R. Coura ${ }^{1}$
}

COURA, J. R. The False Dilemma about Antivectorial Strategies and Possibilities for Controlling Chagas' Disease in Brazil. BHC or BNH? Cad. Saúde Públ., Rio de Janeiro, 9 (4): 514-518, Oct/Dec, 1993.

The technical basis for the control of Chagas' disease in Brazil was established with the creation of the Oswaldo Cruz Institute's research center in Bambui, in western Minas Gerais, at the beginning of the 1940's, under the leadership of Emmanuel Dias. However, only with the creation of the National Department for Rural Endemic Diseases, in March 1956, did control of rural endemic diseases become systematic in Brazil.

From the 1960's onwards, the Chagas' disease control program in Brazil started to suffer from postponements and lack of systematic action: first because the priority ascribed to the malaria control program, since this was an acute, "explosive" disease that was hampering land settlement and development in the interior; second the "new ecologists" began calling for "BNH" (referring to the initials for the national low-cost housing program) and not "BHC" (insecticides program) for Chagas' disease control, ignoring a number of issues and difficulties; and third, other priorites given to the Special Program for Schistosomiasis Control and the meningococcal meningitis epidemic in the 1970's and the dengue epidemic in the 1980's.

In 1983, however, a new phase in Chagas' disease control began, with the allocation of ten billion cruzeiros from FINSOCIAL for this program which covered two thousand municipalities, in 19 Brazilian States, to the benefit of 47 million people potentially at risk.

Finally it was concluded that in the short term, insecticide spraying is the most effective and manageable control measure against natural transmission of Chagas' infection. Other methods of control and urbanization of Chagas' disease are also discussed.

Key words: Chagas' Disease; Vector Control; Insecticides; Urbanization

Em 1948, o conceituado pesquisador mineiro José Pellegrino, após ter concluído com sucesso experiências de campo com o BHC (hexaclorociclo-hexano) no controle de vetores da doença de Chagas, na localidade de Água Comprida, Minas Gerais, passou um famoso telegrama ao então Ministro da Saúde, nos seguintes termos: "Controle da doença de Chagas tecnicamente resolvido".

\footnotetext{
${ }^{1}$ Departamento de Medicina Tropical do Instituto Oswaldo Cruz da Fundação Oswaldo Cruz. Avenida Brasil 4.365, Rio de Janeiro, RJ, 21045-000, Brasil.
}

Passados 45 anos, o que era "tecnicamente resolvido" não foi politicamente solucionado, apesar dos esforços que vêm sendo desenvolvidos nos últimos anos (Fiusa-Lima, 1983; Dias, 1986). Várias dificuldades de ordem econômica, política, social e, até, ecológica foram pretextos para o adiamento do controle da doença de Chagas no Brasil.

As bases técnicas para o controle da doença de Chagas no Brasil foram estabelecidas a partir da instalação do posto do Instituto Oswaldo Cruz em Bambuí, oeste de Minas Gerais, no início da década de 40, sob a orientação de Emmanuel Dias. Os trabalhos pioneiros de dias (1945) e Dias \& Pellegrino (1948), em Minas 
Gerais, e de Freitas (1946) e Freitas et al. (1959) em São Paulo, estabeleceram essas bases. Entretanto, até o advento do governo de Juscelino Kubitschek, em 1956, os trabalhos ficaram limitados a experimentos de campo localizados. Somente com a criação do Departamento Nacional de Endemias Rurais, em março de 1956, com Mario Pinotti, e depois de sua ascenção ao Ministério da Saúde, iniciou-se, de forma regular, o controle das "endemias rurais" no Brasil.

As 12 "campanhas" de controle das endemias rurais, inclusive a doença de Chagas, dividiam o "bolo" orçamentário, que embora de certa forma alentado, não era suficiente para a cobertura completa de todas elas, cabendo naturalmente à malária, pelo seu impacto de doença aguda "explosiva", que dificultava o desbravamento do interior, a maior parcela, ficando a doença de Chagas e as outras endemias em segundo plano.

Dificuldades de diversas naturezas foram postergando ou tornando irregular a campanha de controle da doença de Chagas no Brasil a partir da década de 60, inclusive, e com grande ênfase, pela voz pouco esclarecida dos "novos ecologistas", que diziam "precisamos de BNH (Banco Nacional de Habitação), não de BHC", ignorando fatos importantes como: 1) a inviabilidade econômica de uma ação ampla do BNH, ou seja, da construção de casas, a nível nacional, a curto prazo; 2) a possibilidade de invasão, pelos triatomíneos, das casas, mesmo as de melhor padrão, se a população não estivesse suficientemente educada para combatê-los; e 3) a ação eficaz dos inseticidas residuais no controle dos triatomíneos, cujo emprego em saúde representa menos de 5\% do total empregado no país, a maior parte na agricultura.

Finalmente, a falta de prioridade dada ao controle da doença de Chagas atrasou este controle em pelo menos duas décadas. $\mathrm{Na}$ década de 60, o atraso deveu-se aos motivos acima apontados; na de 70, aos desvios da Superintendência de Campanhas (Sucam) para o controle da epidemia de meningite meningocócica e à prioridade dada ao Programa Especial de Controle de Esquistossomose (Pece). Em 1983, uma nova fase foi aberta para o controle da doença de Chagas, quando o Banco Nacional de Desenvolvimento Econômico e Social
(BNDES), destinou 10 bilhões de cruzeiros, provenientes do Finsocial (Contribuição para financiamento social), para a cobertura de 2.000 municípios de 19 Estados brasileiros, beneficiando 47 milhões de pessoas expostas. Infelizmente, mais uma vez o programa vem sendo desvirtuado, em parte pelo deslocamento de pessoal para o controle de epidemia de dengue, nos últimos anos.

\section{CONTROLE DE VETORES DOMICILIADOS}

Pelo menos 42 espécies de triatomíneos potencialmente vetores do Trypanosoma cruzi já foram assinaladas no Brasil (Lent \& Wygodzinsky, 1979). Entretanto, apenas algumas espécies têm importância na transmissão da infecção ao homen em nosso país, entre os quais destacam-se o Triatoma infestans, o Panstrongylus megistus e os Triatomas brasiliensis, sordida e pseudomaculata.

O T. infestans, a mais domiciliar das espécies de importância epidemiológica no Brasil, tem sido o principal alvo das campanhas de controle, pela sua larga distribuição geográfica, grande antropofilia e, principalmente, pela sua total domiciliação em nosso país, que o torna o princial e o mais vulnerável dos vetores da infecção chagásica. Segue-se em importância o P. megistus, domiciliado em algumas áreas, porém com uma grande importância estratégica, dados o seu caráter domiciliar e silvestre, grande capacidade de reinvasão domiciliar (Forattini et al., 1983a), algumas vezes alternando com o $T$. infestans, capacidade vetorial e dificuldade de controle. Os T. brasiliensis, sordida e pseudomaculata, o primeiro restrito ao Nordeste brasileiro, têm uma grande importância pelo seu caráter ubiqüitário, vivendo no peri e intradomicílio e também possuindo uma grande capacidade de reinvasão domiciliar, como é o caso do sordida, o mais importante dos "vetores secundários" (Forattini, 1980; Forattini et al., 1983b).

Apesar das dificuldades das campanhas de controle da transmissão vetorial da doença de Chagas, mencionadas na introdução deste trabalho, o Brasil é certamente o mais bem sucedido dos países do continente neste sentido. 
Em primeiro lugar, por não ter sido ainda detectada resistência dos triatomíneos aos inseticidas residuais; em segundo lugar, pelos exemplos efetivos de controle, como no Estado de São Paulo, onde a Superintendência de Campanhas Contra Endemias (Sucen) considerou controlada a transmissão da infecção na década de 70, utilizando, hoje, quantidades mínimas de inseticidas em seu programa de vigilância. No Brasil como um todo, de 1984 a 1989, com o programa financiado pelo Finsocial, houve uma extensa cobertura profilática, com expressiva redução do T. infestans, colocando grande número de municípios em fase de vigilância (Dias, 1987, 1991).

Classicamente, as campanhas de controle de vetores com inseticidas residuais se desenvolvem em quatro fases: a) Preparatória: informações epidemiológicas prévias, recrutamento e treinamento de pessoal, reconhecimento geográfico e cadastramento de prédios, informação à população alvo; b) ataque: expurgo maciço em "arrastão", com cobertura total de áreas contíguas, seguido de rociado seletivo 60 a 90 dias depois nos domicílios e peridomicílios residualmente infestados; c) Vigilância entomológica: busca ativa de triatomíneos nas áreas trabalhadas, com rociamento seletivo dos domicílios e peridomicílios nos quais foram encontrados triatomíneos; e d) Avaliação dos resultados: levantamentos entomológicos e sorológicos, principalmente dos nascidos depois do expurgo.

Os inseticidas mais freqüentemente utilizados no Brasil são os "piretróides" e o BHC (hexacloro-ciclo-hexano), este último na dose mínima de 0,5 gramas do isômero gama por metro quadrado, o qual tem uma alta ação inseticida inicial, poder residual de 30 a 60 dias e reduzida ação tóxica para o homem, quando bem utilizado e corretamente manipulado. O BHC é relativamente barato, porém o seu baixo poder residual aumenta o custo operacional pela necessidade de rociamentos mais freqüentes. Os piretróides, principalmente o dieldrin, embora tenham uma ação inicial menor que o BHC, quando aplicados na dose de um grama do produto ativo por metro quadrado, têm uma ação residual de vários meses, equilibrando o seu preço com o BHC, pela redução do custo operacional. Além do elevado custo, o dieldrin é altamente tóxico para operadores e animais domésticos.

Apesar dos inconvenientes e de sua ação transitória, necessitando uma permanente vigilância pela possibilidade de reintrodução ou invasão de vetores secundários, os inseticidas residuais ainda representam a mais importante arma, a curto prazo, para o controle da transmissão natural da infecção chagásica ao homem (Rocha e Silva, 1979; Dias, 1986). Em trabalho que desenvolvemos em áreas de campo no oeste de Minas Gerais (Coura et al., 1984), verificamos que, 15 anos depois do início da borrifação com o BHC, embora irregular, houve uma redução de 65 vezes na prevalência sorológica da infecção chagásica em menores de 15 anos em relação à população geral, reduzindo-se a praticamente zero a transmissão da infecção.

Finalmente, concluímos que o BHC, a curto prazo, é a medida mais eficaz e exequível no controle da transmissão natural da infecção chagásica, devendo-se estimular, a médio e longo prazos, o BNH - construção de casas adequadas e em número suficiente para a população - , acompanhado da educação de base, única forma de prevenção permanente desta e de outras endemias.

Os métodos alternativos de controle biológico, esterilização induzida, inibidores do crescimento, feromônios e atrativos ainda são especulativos e experimentais.

\section{DOENÇA DE CHAGAS COMO ENDEMIA URBANA}

O problema da Doença de Chagas já não se limita mais às zonas rurais endêmicas, interessando cada vez mais aos grandes centros urbanos que recebem a migração das populações do interior à procura de melhores condições de vida, por motivos de ordem econômica, social e particular, inclusive por motivos de saúde.

Embora as causas individuais sejam as mais diversas, é naturalmente o fator econômico aquele que orienta o sentido das correntes migratórias mais volumosas das zonas rurais, de infra-estrutura precária, para os centros urbanos industrializados. 
A abertura das vias de comunicação terrestre, dando maior acesso ao homem do interior que, inconformado com as suas precárias condições de subsistência, se transfere para as grandes cidades, à procura de melhores oportunidades —, vem modificando, dia a dia, o caráter regional das chamadas endemias rurais, cada vez mais freqüentes nos grandes centros urbanos.

Um estudo de revisão feito por Schlemper Jr. (1975), e complementado por Dias (1979) sobre 53 inquéritos sorológicos realizados de 1947 a 1977 por vários autores brasileiros, envolvendo mais de 360 mil candidatos a doadores de sangue de diversas áreas urbanas de nove estados do Brasil, revelou uma prevalência sorológica que variou de zero a $25,8 \%$, com uma mediana de $5,3 \%$ de soros positivos para a infecção chagásica. Esta mediana é muito próxima da média de positividade sorológica encontrada pela Sucam no inquérito nacional realizado entre 1975 e 1980 no interior do país.

Considerando que dois terços da população brasileira vivem hoje em zonas urbanas, é lícito supor que existe, globalmente, um maior número de chagásicos vivendo nessas áreas do que nas zonas rurais, embora proporcionalmente $\mathrm{o}$ percentual seja mais elevado nestas últimas. Se considerarmos um percentual médio de $1,5 \%$ de indivíduos sorologicamente positivos para a infecção chagásica nas zonas metropolitanas do Rio de Janeiro e São Paulo, com mais de 21 milhões de habitantes, teremos 315 mil portadores da infecção, o equivalente à população de uma cidade brasileira de médio porte.

O número de portadores da infecção chagásica nos centros urbanos sofre pequenas oscilações anuais, para mais ou menos, com as migrações, casos que adquirem a infecção por transfusão de sangue, mortes, etc., podendo esta ser perfeitamente caracterizada como uma verdadeira "endemia urbana".

Três aspectos devem ser considerados no problema da doença de Chagas nos grandes centros urbanos: o primeiro se refere ao paciente como entidade mórbida, com suas manifestações clínicas e psicossomáticas diante das novas condições ambientais que tem de enfrentar; o segundo diz respeito aos aspectos sociais e trabalhistas do novo habitante da metrópole; e o terceiro, de responsabilidade médico-sanitá- ria, é o paciente como elo de uma cadeia epidemiológica artificial, candidato potencial a doador de sangue.

\section{RESUMO}

COURA, J. R. O Falso Dilema sobre a Luta Antivetorial e as Perspectivas de Controle da Doença de Chagas no Brasil. BHC ou BNH? Cad. Saúde Públ., Rio de Janeiro, 9 (4): 514-518, out/dez, 1993.

As bases técnicas para o controle da doença de Chagas no Brasil foram estabelecidas com a criação do posto avançado de pesquisa do Instituto Oswaldo Cruz em Bambuí, no oeste de Minas Gerais, no começo da década de 40, sob a liderança de Emmanuel Dias.

Entretanto, somente com a criação do Departamento Nacional de Endemias Rurais (DNERu), em março de 1956, sob a direção de Mario Pinotti, no governo de Juscelino Kubitschek, as medidas de controle foram implementadas.

Das "campanhas" de controle das 12 endemias rurais estabelecidas pelo DNERu, a malária, pelo seu caráter de doença aguda e explosiva, sempre teve a maior parte orçamentária. A doença de Chagas e as outras endemias foram sempre relegadas a um plano secundário de prioridade. Por outro lado, a partir da década de 60, os "novos ecologistas" passaram a criticar o uso de inseticidas, com o slogan de que para controlar a doença de Chagas era necessário o BNH (construção de casas), e não o BHC (uso de inseticidas). Esta opinião, embora equivocada para o controle a curto prazo, teve uma enorme influência negativa sobre o controle dos vetores domiciliados. Apesar disso, algum progresso foi feito neste sentido.

Na década de 70, a epidemia de meningite meningocócica e a priorização do Programa Especial de Controle da Esquistossomose (PECE), pelo Ministro Almeida Machado, com deslocamento de verbas e de pessoal da Superintendência de Campanhas (Sucam) para esses programas, atrasaram ainda mais o controle da doença de Chagas. Somente na década de 80 , com a decisão política do 
Banco Nacional de Desenvolvimento Econômico e Social (BNDES) de alocar 10 bilhões de cruzeiros do Finsocial, o programa de controle da doença de Chagas teve um importante desenvolvimento em 2.000 municípios de 19 Estados brasileiros. Mais uma vez o programa sofre um atraso nesta década, com o deslocamento do pessoal da Sucam para a campanha contra a epidemia da dangue.

Finalmente, concluímos que, a curto prazo, o uso de inseticidas é a medida mais efetiva para o controle da transmissão natural da doença de Chagas. Discutimos também, nesta revisão, as outras medidas de controle e a urbanização da doença.

Palavras-Chave: Doença de Chagas; Controle de Vetores; Inseticidas; Urbanização

\section{REFERÊNCIAS BIBLIOGRÁFICAS}

COURA, J. R.; ABREU, J. L.; DUBOIS, L. E. G.; CORREIA-LIMA, F; ARRUDA JUNIOR, E.; WILLCOX, H. P. F.; ANUNZIATO, N. \& PETANA, W., 1984. Morbidade da doença de Chagas. II. Estudos seccionais em quatro áreas de campo no Brasil. Memórias do Instituto Oswaldo Cruz, 79: 101-124

DIAS, E., 1945. Um Ensaio de Profilaxia da Doença de Chagas. Rio de Janeiro: Imprensa Nacional.

DIAS, E. \& PELLEGRINO, J., 1948. Alguns ensaios com o gammexane no combate aos transmissores da doença de chagas. Brasil Médico, 62: 185191.

DIAS, J. C. P., 1986. O programa de controle da doença de Chagas no Brasil em 1986. Revista da Sociedade Brasileira de Medicina Tropical, 19: 129-133.

1987. Control of Chagas ' disease in Brazil. Parasitology Today, 3: 336-341.

, 1979. Mecanismos de transmissão. In: Trypanosoma cruzi e Doença de Chagas ( $Z$. Brener \& Z. Andrade, eds.), pp. 152-174, Rio de Janeiro: Guanabara Koogan.

, 1991. O controle da doença de Chagas no Brasil. Revista da Sociedade Brasileira de M edicina Tropical, 24 (Supl. 1): 201-202.
FIUSA-LIMA, J. T., 1983. Incremento do programa de controle da doença de Chagas no Brasil. Revista da Sociedade Brasileira de Medicina Tropical, 16: 128-129.

FORATTINI, O. P., 1980. Biogeografia, origem e distribuição da domiciliação de triatomíneos no Brasil. Revista de Saúde Pública, 14: 265-299.

FORATTINI, O. P.; FERREIRA, O. A.; RABELLO, E. X.; BARATA, J. M. \& SANTOS, J. L. F., 1983a. Aspectos ecológicos da tripanossomíase americana. XVII. Desenvolvimento da domiciliação triatomínea regional em centro de endemismo de Triatoma sordida. Revista de Saúde Pública, 17: 159-199.

, 1983b. Aspectos ecológicos da tripanossomíase americana. XIX. Desenvolvimento da domiciliação triatomínea regional em centro de endemismo da Panstrongylus megistus. Revista de Saúde Pública, 17: 436-460.

FREITAS, J. L. P., 1946. Inquérito preliminar sobre doença de Chagas no município de Cajuru, Estado de São Paulo. Hospital, 29: 155-165.

FREITAS, J. L. P.; FERREIRA, D. A.; GARCIA, G. \& HADDAD, N., 1959. Resultado do combate intenso dos triatomíneos domiciliares em uma área restrita do Estado de São Paulo (distrito de Cassia de Coqueiros, município de Cajuru). In: A nais do Congresso Internacional sobre Doença de Chagas, vol. II, pp. 544-556, Rio de Janeiro.

LENT, H. \& WYGODZINSKY, P., 1979. Revision of the triatominae (Hemiptera, reduvidae) and their significance as vectors of Chagas' disease. Bulletin of American Museum of $\mathrm{N}$ atural History, 520p.

ROCHA E SILVA, E. O., 1979. Profilaxia. In: Trypanosoma cruzi e Doença de Chagas (Z. Brener \& Z. Andrade, eds.), pp. 425-449, Rio de Janeiro: Guanabara Koogan.

SCHLEMPER JR., B. R., 1975. Estudos Experimentais e Quimioprofilaxia da Transmissão da Doença de Chagas por Transfusão Sangüinea. Tese de Mestrado, Belo Horizonte: Universidade Federal de Minas Gerais. 